\title{
Application of three-dimensional image reconstruction technology based on high-resolution CT in pyeloplasty
}

\author{
Xuechao Li ${ }^{1,2 \#}$, Jingyun Zhang ${ }^{2 \#}$, Weiqing Shi ${ }^{2 \#}$, Tao Yang ${ }^{2}$, Rongjian Lu$^{2}$, Xiaoling Zhao ${ }^{3}$, Lijun Chen ${ }^{2}$ \\ ${ }^{1}$ Medical School of Chinese PLA, Beijing, China; ${ }^{2}$ Fifth Medical Centre of Chinese PLA General Hospital, Beijing, China; ${ }^{3}$ Cheerland Clinical \\ Laboratory Co., Ltd., Beijing, China \\ Contributions: (I) Conception and design: X Li; (II) Administrative support: L Chen; (III) Provision of study materials or patients: J Zhang; (IV) \\ Collection and assembly of data: W Shi, T Yang; (V) Data analysis and interpretation: X Li, R Lu, X Zhao; (VI) Manuscript writing: All authors; (VII) \\ Final approval of manuscript: All authors. \\ \#These authors contributed equally to this work and should be considered co-first authors. \\ Correspondence to: Lijun Chen. Fifth Medical Centre of Chinese PLA General Hospital, Beijing 100071, China. Email: chenlj829@163.com.
}

Background: Three-dimensional (3D) image reconstruction technology is widely used in surgical operations for its intuitive visualization. Pyeloplasty requiresprecise cutting and suturing. The reconstruction technology can accurately determine the location and scope of the stenosis at the junction of the renal pelvis and ureter and the relationship with the surrounding vasculature. The purpose of this article is to retrospective evaluate the application value of image reconstruction technology in pyeloplasty based on highresolution 3D CT images.

Methods: A total of 20 patients with renal pelvic ureteral junction obstruction admitted to our hospital from August 2019 to August 2020 were selected. In this group, left pyeloplasty was performed in 8 patients and right pyeloplasty in 12 patients. In terms of conditions, there was 1 case with secondary pyeloplasty, 6 cases of patients with kidney stones, 2 cases with renal ectopic blood vessels, 1 case with renal prolapse, 1 case with horseshoe kidney, and 1 case with ureteral polyps. There were 12 males and 8 females, with an average age of $34.65 \pm 10.67$ years and an average body mass index (BMI) of $22.48 \pm 3.03 \mathrm{~kg} / \mathrm{m}^{2}$. In all patients, 3D CT reconstruction technology was used to guide the formulation of robot-assisted laparoscopic pyeloplasty plans; verify the consistency between the actual operation and the preoperative planning; and observe the operation time, blood loss, postoperative exhaust time, indwelling drainage tube time, and follow-up for comorbidities. Results: The operation was successful in all 20 patients. The actual operation was $100 \%$ consistent with the preoperative planning, the operative time was $160.80 \pm 63.26 \mathrm{~min}$, the intraoperative blood loss was $47 \pm 30.45 \mathrm{~mL}$, the postoperative exhaust time was $1.15 \pm 0.37$ days, the drainage tube indwelling time was $4.35 \pm 1.50$ days, and the average follow-up time was $7.95 \pm 3.41$ months. There were no complications.

Conclusions: Three-dimensional image reconstruction technology based on high-resolution CT has high clinical application value in the treatment of ureteropelvic junction obstruction (UPJO), which simplifies the operation process and shortens the operation time, and is a valuable tool for auxiliary surgeons in devising the operation plan.

Keywords: Three-dimensional image reconstruction technology (3D image reconstruction technology); robotassisted laparoscopy; pyeloplasty

Submitted Dec 31, 2020. Accepted for publication Mar 18, 2021.

doi: $10.21037 /$ tau-21-202

View this article at: http://dx.doi.org/10.21037/tau-21-202 


\section{Introduction}

Ureteropelvic junction obstruction (UPJO) is common and mostly congenital. Dysplasia of smooth muscle fibers at the ureteropelvic junction is more common, resulting in stenosis of the site or conduction and peristaltic dysfunction. It causes hydronephrosis. Long-term obstruction often leads to the formation of secondary renal stones, and also increases the risk of urinary tract infection, renal parenchymal atrophy, and renal function impairment (1). Since 1949 (2), Anderson-Hynes disengaged pyeloplasty has been the most accepted and used method for UPJO (3), with an effective rate of over $91.1 \%$ (4). High-resolution computed tomography (CT) scans can clearly show the hydronephrosis, ureteropelvic junction (UPJ) location, and the comprehensive shape; however, the information concerning the exact location and anatomical space is not ideal. Three-dimensional (3D) reconstruction technology can present the complete anatomical morphology and spatial relationship. Combined with robot-assisted laparoscopic technology, it realizes the perfect integration of digital with precision medicine, representing a breakthrough in treatment for preoperative planning and intraoperative navigation. A total of 20 patients with UPJO admitted to our hospital from August 2019 to August 2020 were retrospectively analyzed to explore the application value in pyeloplasty of 3D image reconstruction technology based on high-resolution CT.

We present the following article in accordance with the STROBE reporting checklist (available at http://dx.doi. org/10.21037/tau-21-202).

\section{Methods}

All procedures performed in this study involving human participants were in accordance with the Declaration of Helsinki (as revised in 2013). This study was approved by the Ethics Committee of the Fifth Medical Centre, Chinese PLA General Hospital (No. 2020-12-20-1). Individual consent for this retrospective analysis was waived.

\section{Clinical data}

The participants in this study were UPJO patients who had undergone surgical operation at the Fifth Medical Centre of Chinese PLA General Hospital, (Beijing, China). In all, 20 UPJO patients were collected from August 2019 to August 2020, and all patients were diagnosed by
Table 1 Basic data of surgical patients

\begin{tabular}{lc}
\hline Patients $(\mathrm{N}=20)$ & Results \\
\hline Male & $12(60 \%)$ \\
Female & $8(40 \%)$ \\
Age (years) & $34.65 \pm 10.67$ \\
BMl (kg/m $\left.{ }^{2}\right)$ & $22.48 \pm 3.03$ \\
Left side & $8(40 \%)$ \\
Right side & $12(60 \%)$ \\
With kidney stones & $6(30 \%)$ \\
With ectopic vessels & $2(10 \%)$ \\
With horseshoe kidney & $1(5 \%)$ \\
With renal prolapse & $1(5 \%)$ \\
With ureteral polyps & $1(5 \%)$ \\
Secondary pyeloplasty & $1(5 \%)$ \\
\hline
\end{tabular}

intravenous urography angiography and CT examination. Eight patients had left side surgery and 12 patients had right side surgery. There were 12 male and 8 female patients, with an average age of $34.65 \pm 10.67$ years and an average body mass index (BMI) of $22.48 \pm 3.03 \mathrm{~kg} / \mathrm{m}^{2}$. There was 1 case with the secondary pyeloplasty, 6 cases with kidney stones, 2 cases with kidney ectopic vessel, 1 case with renal prolapse, 1 case with horseshoe kidney, and 1 case with ureteral polyps. The detailed patient data are summarized in Table 1.

\section{$3 D$ reconstruction and surgical methods}

All 20 UPJO patients underwent a 64-slice spiral CT enhanced thin-slice preoperative scan, including a plain scan, arterial scan, venous scan, and delayed scan. The original CT data were collected and stored in DICOM (Digital Imaging and Communications in Medicine) data format. The DICOM data of the patients in each stage were imported into a medical image processing software image processing system (IPS) (version 2.0.7.0, Shenzhen Xudong Digital Medical Imaging Technology Co., Ltd., China) for 3D reconstruction. The UPJO 3D model was established to observe the stenosis of the UPJ and the location of its adjacent blood vessels and intestines from multiple angles and included visual manipulations, such as amplification and rotation, to allow for precise preoperative planning and selection of the surgical method (Figures 1-3). 


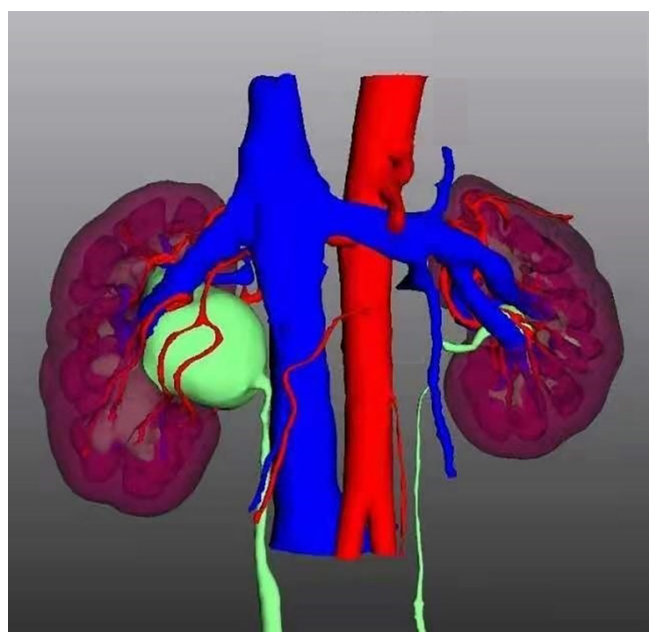

Figure 1 UPJO 3D imaging. UPJO, ureteropelvic junction obstruction; 3D, three-dimensional.

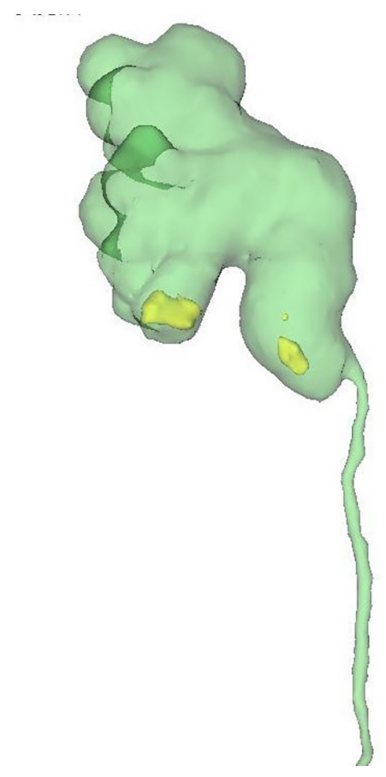

Figure 2 The UPJO with kidney stones. UPJO, ureteropelvic junction obstruction.

\section{Surgical steps}

According to the results of preoperative CT examination and $3 \mathrm{D}$ image reconstruction, the surgical plan was established, and robot-assisted laparoscopic pyeloplasty was performed in all 20 patients. The method of anesthesia was general anesthesia via endotracheal intubation. The patient assumed a $45^{\circ}$ oblique position on the healthy side, padded at the waist. Flex the table such that there is slight extension

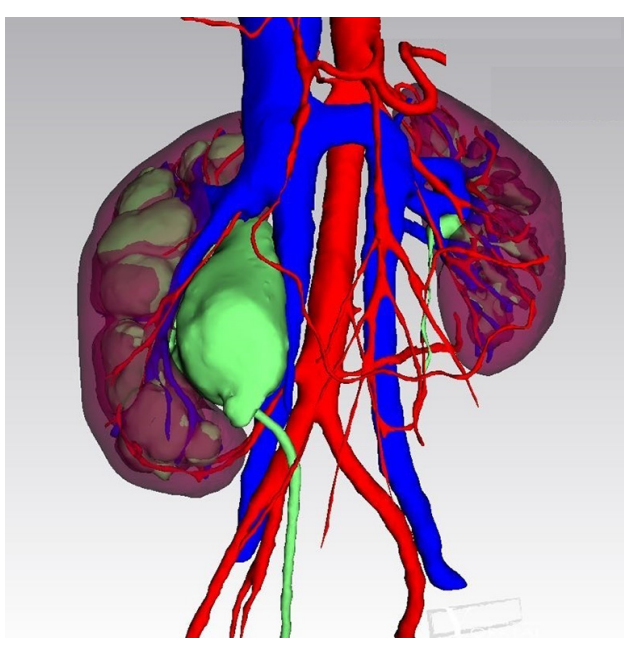

Figure 3 The UPJO with ectopic arterial vessels. UPJO, ureteropelvic junction obstruction.

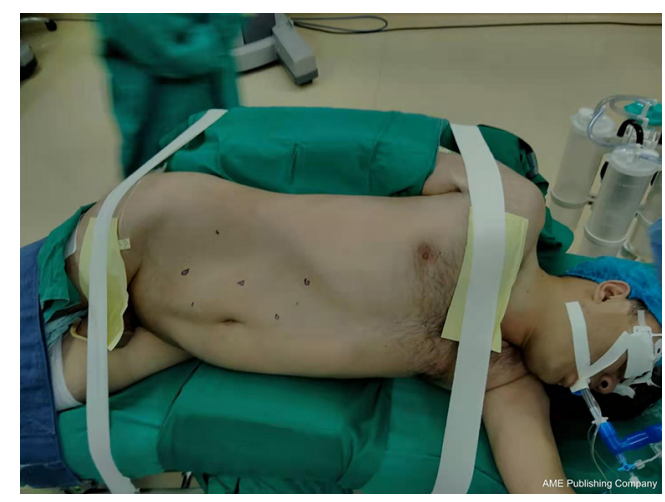

Figure 4 Design of robotic surgical approach.

of the flank.

The healthy side of the upper limb was placed on the side of the body to be fixed on the arm frame, while the affected side of the upper limb was placed on the side of the body to be fixed. The patient is disinfected in the surgical area and placed with a sterile drape. The robot-assisted laparoscopic port is designed as follows (Figure 4, taking the right lesion as an example): $12 \mathrm{~mm}$ lens port: the outer edge of the affected side of the rectus abdominal muscle was flat at the level of stenosis at the junction of the renal pelvis and ureter, according to $3 \mathrm{D}$ image reconstruction; the first $8 \mathrm{~mm}$ manipulator special port: the distance from the lens port below the outer edge of the affected side rectus abdominal muscle was $8 \mathrm{~cm}$; the second $8 \mathrm{~mm}$ special port for the robotic arm: take a distance of $8 \mathrm{~cm}$ on the line 
Table 2 Surgery and follow-up results

\begin{tabular}{lc}
\hline Observation item & Results \\
\hline Surgical success rate & $100 \%$ \\
Operation time & $160.80 \pm 63.26$ minutes \\
Intraoperative blood loss & $47 \pm 30.45 \mathrm{~mL}$ \\
Postoperative exhaust time & $1.15 \pm 0.37$ days \\
Drainage tube indwelling time & $4.35 \pm 1.50$ days \\
Follow-up time & $7.95 \pm 3.41$ months \\
Complications & None \\
\hline
\end{tabular}

where the angle between the first arm port and the lens port is $120^{\circ}$; the third $8 \mathrm{~mm}$ special port for robotic arm: this was $2 \mathrm{~cm}$ from the pubic symphysis on the lateral edge of the rectus abdominis. The $12 \mathrm{~mm}$ assistant port was located $6 \mathrm{~cm}$ on the vertical line between the lens port and the first mechanical arm port. The vertical line was $6 \mathrm{~cm}$ from the midpoint between the lens port and the third arm port, being used with the $5 \mathrm{~mm}$ second assistant port. The second assistant port was established according to the difficulty of the operation. The first arm used monopolar curved scissors, the second arm used Maryland forceps bipolar, and the third arm used prograsp forceps. The lateral peritoneum was incised at the paracolic sulcus with monopolar curved scissors, and the colon was dissociated inwards and downwards to expose the retroperitoneal space. To find the expansion of the renal pelvis and UPJ position, free renal pelvis and upper ureter (should be dissected if accompanied by ectopic vessels). The renal pelvis was cut above UPJ and at the lower end of the renal pelvis, with a length of $1.0 \mathrm{~cm}$. Split the ureter vertically downward to $1.5 \mathrm{~cm}$ below the UPJ. Trim the renal pelvis and cut off the ureter. If patients have renal calculi, flexible cystoscope should be performed at the same time to assist with pyelolithotomy. The first needle was sutured with a 4-0 absorbable thread at the lower pole of the renal pelvis incision and the lower corner of the ureter. The posterior wall of the ureteropelvis anastomosis was continuously sutured, and a 7F $26-\mathrm{cm}$ ureteral stent was indwelling under the guide wire.

The anterior wall of the ureteropelvic anastomosis was continuously sutured with 4-0 absorbable suture, and the renal pelvis incision was closed with a continuous suture.

\section{Observation index}

The general condition of the patient was analyzed and included the verification of the consistency of actual operation and preoperative planning, operation time, intraoperative blood loss, postoperative exhaust time, drainage tube indwelling time, average follow-up time, and complications. The data were processed by SPSS 24.0 software (IBM Corp., USA), and the measurement data are expressed as mean \pm standard deviation.

\section{Results}

All 20 patients successfully underwent surgical treatment. The actual operation was consistent with the preoperative plan. There was no transfer or opening, and the surgical success rate was $100 \%$. The operation time was $160.80 \pm 63.26$ minutes, intraoperative blood loss was $47 \pm 30.45 \mathrm{~mL}$, time to postoperative exhaust was $1.15 \pm 0.37$ days, drainage tube indwelling time was $4.35 \pm 1.50$ days, follow-up time was $7.95 \pm 3.41$ months, and no complications were found (see Table 2 for details).

\section{Discussion}

UPJO is a relatively common urinary tract obstructive disease in urology. It is mostly congenital and can appear at any age with many clinical symptoms, such as back pain, abdominal mass, hematuria, etc. The most classic surgical method for simple UPJO is Anderson-Hynes dissected pyeloplasty. With the development of minimally invasive technology, laparoscopic pyeloplasty and robot-assisted laparoscopic pyeloplasty are gradually being accepted by most clinicians over open surgery (5). Due to the complicated anatomical structure of the renal hilum area and the narrow space, the ureteral stenosis is closely related to the adjacent dilated renal pelvis, renal artery, renal vein, reproductive vein, abdominal aorta, inferior vena cava and duodenum, which increases the risk in UPJO operations. Therefore, the goal of precision medicine is determining how to accurately remove the lesion, reduce the disturbance to healthy tissues, and minimize surgical complications, toward achieving "the smallest trauma, the largest organ protection and the best rehabilitation effect" (6). In recent years, due to the rapid development of $3 \mathrm{D}$ visualization technology, precision surgical lithotripsy has been increasingly improved (7). By means of fullangle rotation of stereoscopic image, the image can be zoomed, transparent, and wiped out of specific organs and tissues. Specific organs, tissues or blood vessels can be arbitrarily split, combined, hidden or displayed according 
to needs, and the size, location and stereoscopic structural relationship between the lesion and surrounding tissues and organs can be studied. The 3D images constructed preoperatively are tracked, projected and fused in real time with the operative field images in the laparoscopic monitor through specific software, so as to realize intraoperative navigation, assist the operator in intraoperative positioning of vital organs, blood vessels and lymph nodes, and finely guide the operation. Through $3 \mathrm{D}$ reconstruction technology, physicians can measure the volume of hydronephrosis through the software before surgery (8), and observe the specific position and precise length of ureteral stricture through multiangle rotation, showing the shape of the ureter and the relationship between the ureteral stenosis and the vessel, which can simulate the cutting range, adjust the surgical plan, and improve the safety of the operation (9). In this study, 20 patients underwent $3 \mathrm{D}$ reconstruction and simulated surgical planning before the operation. During the operation, the operation was performed according to the preoperative planning. The operation was smooth, reduced the disturbance to tissues and organs outside the surgical target area, and avoided surrounding tissues. The accuracy of the operation involves avoiding organ damage and ensuring patient safety. The $3 \mathrm{D}$ reconstruction model provides the surgeon with a greater perceptual understanding of the operation content, allowing, for example, for the surgeon to judge the relationship between the patient's ectopic blood vessel and UPJ and the blood supply range to the kidney. Most renal ectopic blood vessels are derived from the renal artery, abdominal aorta, inferior vena cava, and iliac vessels, which supply the lower pole of the kidney. The incidence of renal heterotopic blood vessels in adults is about $20 \%$, and the incidence of UPJO patients is about $38-71 \%$ (10). In this study, 3D image reconstruction was used to clearly show that 2 UPJO patients had ectopic subrenal vessels: in one case, the artery originated from the abdominal aorta and extended behind the UPJ, in the other case, the vein originated from the inferior vena cava and traveled in front of the UPJ. When ectopic blood vessels are arteries, they should be preserved. When they are difficult to avoid, the blood vessels are thin, and the blood supply to the kidney is small, they can be cut off. Because of the presence of urine stasis and infection, crystals in the urine are deposited and aggregate, increasing the likelihood of stone formation. The incidence of UPJO accompanied by stones is about 20\% (4). For UPJO patients with kidney stones, the stones can be located on the upper, middle, and lower kidneys, with each location being subdivided into front and back areas. It is difficult to judge the exact location of the stones from the 2D CT images. The surgeon can accurately use the 3D reconstruction model to determine the size and location of the stone. In this study, 6 cases of UPJO were accompanied by kidney stones. We used a flexible bladder to enter the renal pelvis through the abdominal assistant port to complete the lithotripsy, which shortens the operation time. Furthermore, based on the preoperative $3 \mathrm{D}$ reconstruction, we can predict the location of the stones and thus reduce the incidental damage to the tissue due to repeated access to the stone removal equipment. Hong et al. (11) reported that compared to the flexible ureteroscope, the flexible cystoscope is easier to control and has a wide field of vision. For UPJO patients with renal ptosis or horseshoe kidney, due to the abnormal kidney position, rotation, and blood vessel supply (12), the anatomy and adjacent position of UPJ will have greater changes than in general UPJO patients, and so surgical planning based on preoperative $3 \mathrm{D}$ image reconstruction is particularly critical for the design and establishment of the robotic surgical port. In one UPJO case with renal ptosis in this study, the position of UPJ was flush with the navel. Therefore, the port was moved down about $2.0 \mathrm{~cm}$ compared to the approach with the general UPJO patients, according to the $3 \mathrm{D}$ reconstruction image. In another UPJO patient with horseshoe kidney, the position of the UPJ was significantly elevated due to improper rotation of the affected side of the kidney. Therefore, the port was designed based on 3D reconstructed images and moved approximately $2.0 \mathrm{~cm}$ to the midclavicular line, as compared with general UPJO patients, which increased the accuracy of the surgical operation. Secondary pyeloplasty is particularly difficult and challenging (13); however, 1 case of secondary pyeloplasty was successfully completed in the study with the help of $3 \mathrm{D}$ reconstruction technology.

This study's operation time (78-368 minutes) was shorter than that that of the robotic pyeloplasty by Tasian et al. (123-384 minutes) (14). The intraoperative blood loss was $62.00 \pm 74.31 \mathrm{~mL}$, which was similar to the average intraoperative blood loss of $50 \mathrm{~mL}$ reported by Habicher et al. (15); the postoperative exhaust time was $1.15 \pm 0.37$ days, and the drainage tube indwelling time was $4.35 \pm 1.50$ days. This indicates that the patient's body can recover quickly after surgery, which is largely a result of our successful navigation based on $3 \mathrm{D}$ reconstruction images during the operation.

In summary, 3D image reconstruction technology based on high-resolution CT for robot-assisted laparoscopic 
UPJO pyeloplasty is conducive to preoperative evaluation, facilitating the surgeon's 3D perceptual cognition, and providing surgical planning for precise UPJO resection and renal pelvic reconstruction. Adoption of this technology has great clinical significance in realizing the maximum resection of the lesion, reducing the damage to the surrounding organs, optimizing the surgical path, shortening the operation time, reducing the amount of intraoperative blood loss, minimizing surgical complications, and accelerating postoperative recovery.

\section{Acknowledgments}

Funding: None.

\section{Footnote}

Reporting Checklist: The authors have completed the STROBE reporting checklist. Available at http://dx.doi. org/10.21037/tau-21-202

Data Sharing Statement: Available at http://dx.doi. org/10.21037/tau-21-202

Conflicts of Interest: All authors have completed the ICMJE uniform disclosure form (available at http://dx.doi. org/10.21037/tau-21-202). The authors have no conflicts of interest to declare.

Ethical Statement: The authors are accountable for all aspects of the work in ensuring that questions related to the accuracy or integrity of any part of the work are appropriately investigated and resolved. This study was approved by the Ethics Committee of the Fifth Medical Centre, Chinese PLA General Hospital (No. 2020-12-201). All procedures performed in this study involving human participants were in accordance with the Declaration of Helsinki (as revised in 2013). Individual consent for this retrospective analysis was waived.

Open Access Statement: This is an Open Access article distributed in accordance with the Creative Commons Attribution-NonCommercial-NoDerivs 4.0 International License (CC BY-NC-ND 4.0), which permits the noncommercial replication and distribution of the article with the strict proviso that no changes or edits are made and the original work is properly cited (including links to both the formal publication through the relevant DOI and the license).
See: https://creativecommons.org/licenses/by-nc-nd/4.0/.

\section{References}

1. Jensen PH, Berg KD, Azawi NH. Robot-assisted pyeloplasty and pyelolithotomy in patients with ureteropelvic junction stenosis. Scand J Urol 2017;51:323-8.

2. Anderson JC, Hynes W. Retrocaval ureter; a case diagnosed pre-operatively and treated successfully by a plastic operation. Br J Urol 1949;21:209-14.

3. Göğüş C, Karamürsel T, Tokatli Z, et al. Long-term results of Anderson-Hynes pyeloplasty in 180 adults in the era of endourologic procedures. Urol Int 2004;73:11-4.

4. Stravodimos KG, Giannakopoulos S, Tyritzis SI, et al. Simultaneous laparoscopic management of ureteropelvic junction obstruction and renal lithiasis: the combined experience of two academic centers and review of the literature. Res Rep Urol. 2014;6:43-50.

5. Bergersen A, Thomas R, Lee BR. Robotic Pyeloplasty. J Endourol 2018;32:S68-S72.

6. Dong J, Zhang N. Precise surgical. Zhonghua Wai Ke Za Zhi 2015;53:321-3.

7. Wang Z, Qi L, Yuan P, et al. Application of ThreeDimensional Visualization Technology in Laparoscopic Partial Nephrectomy of Renal Tumor: A Comparative Study. J Laparoendosc Adv Surg Tech A 2017;27:516-23.

8. Shimoyama $\mathrm{H}$, Isotani $\mathrm{S}$, China $\mathrm{T}$, et al. Automated renal cortical volume measurement for assessment of renal function in patients undergoing radical nephrectomy. Clin Exp Nephrol 2017;21:1124-30.

9. Zheng MM, Ding GP, Zhu WJ, et al. Application of preoperative three-dimensional image reconstruction in the treatment of ureteropelvic junction obstruction. Beijing Da Xue Xue Bao Yi Xue Ban 2020;52:705-10.

10. Ellerkamp V, Kurth RR, Schmid E, et al. Differences between intrinsic and extrinsic ureteropelvic junction obstruction related to crossing vessels: histology and functional analyses. World J Urol 2016;34:577-83.

11. Hong P, Li Z, Zhu D, et al. A Simple Modification for the Usage of Flexible Cystoscope in Modified Laparoscopic Pyeloplasty for Ureteropelvic Junction Obstruction with Renal Calculi: A Flexible Guiding Tube. Urol Int 2019;102:262-8.

12. Kirkpatrick JJ, Leslie SW. Horseshoe Kidney. In: StatPearls. Treasure Island (FL): StatPearls Publishing; 2021.

13. Lee M, Lee Z, Strauss D, et al. Multi-institutional 
Experience Comparing Outcomes of Adult Patients

Undergoing Secondary Versus Primary Robotic

Pyeloplasty. Urology 2020;145:275-80.

14. Tasian GE, Casale P. The robotic-assisted laparoscopic pyeloplasty: gateway to advanced reconstruction. Urol Clin North Am 2015;42:89-97.

15. Habicher M, Tokas T, Herrmann TR, Nagele U; Training and Research in Urological Surgery and Technology (T.R.U.S.T.)-Group. Laparoscopic single-incision triangulated umbilical surgery (SITUS) pyeloplasty: a description of the first 32 cases. World J Urol 2018;36:1883-88.

(English Language Editor: J. Gray)
Cite this article as: Li X, Zhang J, Shi W, Yang T, Lu R, Zhao X, Chen L. Application of three-dimensional image reconstruction technology based on high-resolution CT in pyeloplasty. Transl Androl Urol 2021;10(3):1314-1320. doi: 10.21037/tau-21-202 